\section{FRI0194 INTEGRATED SAFETY PROFILE OF ATACICEPT FROM ALL CLINICAL STUDIES TO DATE}

Caroline Gordon ${ }^{1}$, Roberto Bassi ${ }^{2}$, Peter Chang ${ }^{2}$, Amy Kao ${ }^{2}$, David Jayne ${ }^{3}$, David Wofsy ${ }^{4}$, Victor Ona ${ }^{2}$, Patricia Fleuranceau-Morel ${ }^{2} .{ }^{1}$ University of Birmingham, Birmingham, United Kingdom; ${ }^{2} E M D$ Serono Research and Development Institute, Inc., Billerica, United States of America; ${ }^{3}$ University of Cambridge, Cambridge, United Kingdom; ${ }^{4}$ University of California, San Francisco, United States of America

Background: We conducted an integrated analysis of pooled safety data from all 17 atacicept clinical studies across multiple indications to date. Objectives: To characterize the overall safety profile of atacicept. Methods: Analyses were based on 3 pooled datasets: double-blind placebo (PBO)-controlled set (DBPC-S) ( $n=1568$; key endpoint: treatmentemergent AEs [TEAEs]); systemic lupus erythematosus set (SLE-S; $n=761$; key endpoints: IgG change and serious infection rates, and mortality); and full analysis set (FA-S; $n=1845$; key endpoint: exposureadjusted mortality).

Results: Of 1568 patients (DBPC-S), $30.8 \%$ received PBO, and $8.2 \%$, $24.5 \%$ and $36.5 \%$ received atacicept 25,75 and $150 \mathrm{mg}$. Overall, baseline characteristics were balanced across treatment arms. Treatment exposure in patient-years (py) was similar with PBO and atacicept 75 and $150 \mathrm{mg}$ but was lower with $25 \mathrm{mg}$ (Table). Exposure-adjusted TEAE rates were generally higher with atacicept vs PBO; serious TEAE and infection rates were similar with atacicept and PBO (Table). TEAE-related discontinuation rates were higher with atacicept vs PBO (16.1 vs 10.9/ $100 \mathrm{py}$ ). In the SLE-S and DBPC-S, pharmacodynamic effects of atacicept (eg on $\mathrm{lgG}$ ) were not associated with an increase in infection rates. Across all studies (FA-S), 11 patients died during treatment. Across all indications, exposure-adjusted mortality rates $/ 100$ py $(95 \% \mathrm{Cl})$ were 3.60 $(0.90-14.38), 0.34(0.05-2.43), 1.18(0.49-2.82)$ for atacicept 25,75 and $150 \mathrm{mg}$, and $0.44(0.06-3.12)$ for PBO. In SLE patients, exposureadjusted mortality rates/100 py were $1.45(0.54-3.87)$ with atacicept 150 $\mathrm{mg}$ and $0.78(0.29-2.07)$ across all atacicept-treated patients. The underlying disease and other causes were potential confounders in most cases.

Conclusion: In this integrated safety analysis of atacicept, no consistent association was found between atacicept dose and specific TEAEs or mortality. These results support further development and evaluation of atacicept in patients in whom potential benefits may outweigh risks.

Table.Exposure-adjusted TEAE rates by dose (DBPC-S)

\begin{tabular}{|c|c|c|c|c|c|c|}
\hline & \multirow{2}{*}{$\begin{array}{l}\text { Placebo, } \\
n=483\end{array}$} & \multicolumn{4}{|c|}{ Atacicept } & \multirow{2}{*}{$\begin{array}{c}\text { Total, } \\
n=1568\end{array}$} \\
\hline & & $\begin{array}{l}25 \mathrm{mg}, \\
\mathrm{n}=129\end{array}$ & $\begin{array}{l}75 \mathrm{mg}, \\
\mathrm{n}=384\end{array}$ & $\begin{array}{c}150 \mathrm{mg} \\
\mathrm{n}=572\end{array}$ & $\begin{array}{c}\text { All, } \\
n=1085\end{array}$ & \\
\hline \multicolumn{7}{|l|}{ TEAE, $n$ (rate per 100 py) } \\
\hline Hypersensitivity & 37 (13.9) & $8(15.7)$ & $\begin{array}{c}40 \\
(19.1)\end{array}$ & $55(20.4)$ & $\begin{array}{c}103 \\
(19.4)\end{array}$ & $\begin{array}{c}140 \\
(17.6)\end{array}$ \\
\hline Infections & $\begin{array}{c}211 \\
(107.8)\end{array}$ & $\begin{array}{c}43 \\
(104.4)\end{array}$ & $\begin{array}{c}180 \\
(118.7)\end{array}$ & $\begin{array}{c}281 \\
(141.3)\end{array}$ & $\begin{array}{c}504 \\
(128.7)\end{array}$ & $\begin{array}{c}715 \\
(121.7)\end{array}$ \\
\hline Serious infection & $20(7.3)$ & $1(1.9)$ & $\begin{array}{c}23 \\
(10.5)\end{array}$ & $22(7.7)$ & $46(8.3)$ & $66(7.9)$ \\
\hline Severe infection & $9(3.2)$ & 0 & $11(4.9)$ & $16(5.6)$ & $27(4.8)$ & $36(4.3)$ \\
\hline Herpes zoster & $13(4.7)$ & $2(3.9)$ & $10(4.5)$ & $17(6.1)$ & $29(5.2)$ & $42(5.1)$ \\
\hline Injection site reactions & $54(20.9)$ & $\begin{array}{c}27 \\
(64.8)\end{array}$ & $\begin{array}{c}109 \\
(63.0)\end{array}$ & $\begin{array}{c}156 \\
(72.4)\end{array}$ & $\begin{array}{c}292 \\
(67.9)\end{array}$ & $\begin{array}{c}346 \\
(50.2)\end{array}$ \\
\hline $\begin{array}{l}\text { Severe } \\
\text { hypogammaglobulinemia } \\
(\operatorname{lgG}<3 \mathrm{~g} / \mathrm{L})\end{array}$ & 0 & 0 & $2(0.9)$ & $4(1.4)$ & $6(1.1)$ & $6(0.7)$ \\
\hline Cardiac arrhythmias [all] & $18(6.6)$ & $\begin{array}{c}11 \\
(22.4)\end{array}$ & $\begin{array}{c}23 \\
(10.6)\end{array}$ & $25(8.9)$ & $\begin{array}{c}59 \\
(10.8)\end{array}$ & $77(9.4)$ \\
\hline $\begin{array}{l}\text { Ventricular arrhythmias } \\
\text { Ischemic heart disorders }\end{array}$ & $\begin{array}{c}5(1.8) \\
11(4.0)\end{array}$ & $\begin{array}{c}0 \\
3(5.9)\end{array}$ & $\begin{array}{c}4(1.8) \\
13(5.9)\end{array}$ & $\begin{array}{c}6(2.1) \\
11(3.9)\end{array}$ & $\begin{array}{l}10(1.8) \\
27(4.9)\end{array}$ & $\begin{array}{l}15(1.8) \\
38(4.6)\end{array}$ \\
\hline $\begin{array}{l}\text { Embolic and } \\
\text { thromboembolic events }\end{array}$ & $11(4.0)$ & $1(2.0)$ & $6(2.7)$ & $9(3.2)$ & $16(2.9)$ & $27(3.2)$ \\
\hline Vestibular disorders & $19(7.0)$ & $5(9.9)$ & $18(8.3)$ & $26(9.3)$ & $49(8.9)$ & $68(8.3)$ \\
\hline Demyelination & $1(0.4)$ & $1(1.9)$ & 0 & $5(1.7)$ & $6(1.1)$ & $7(0.8)$ \\
\hline Depression & $14(5.1)$ & $3(5.8)$ & $8(3.6)$ & $11(3.9)$ & $22(3.9)$ & $36(4.3)$ \\
\hline Malignant tumor & 0 & $1(1.9)$ & $1(0.4)$ & $3(1.1)$ & $5(0.9)$ & $5(0.6)$ \\
\hline Serious TEAE & $51(18.9)$ & $\begin{array}{c}15 \\
(30.0)\end{array}$ & $\begin{array}{c}51 \\
(23.9)\end{array}$ & $61(21.8)$ & $\begin{array}{c}127 \\
(23.4)\end{array}$ & $\begin{array}{c}178 \\
(21.9)\end{array}$ \\
\hline Severe TEAE & $28(10.2)$ & $\begin{array}{c}10 \\
(19.6)\end{array}$ & $\begin{array}{c}45 \\
(20.9)\end{array}$ & $56(20.0)$ & $\begin{array}{c}111 \\
(20.3)\end{array}$ & $\begin{array}{c}139 \\
(17.0)\end{array}$ \\
\hline $\begin{array}{l}\text { Discontinuation of } \\
\text { treatment due to TEAE }\end{array}$ & $30(10.9)$ & $\begin{array}{c}14 \\
(27.6)\end{array}$ & $\begin{array}{c}30 \\
(13.4)\end{array}$ & $46(16.1)$ & $\begin{array}{c}90 \\
(16.1)\end{array}$ & $\begin{array}{c}120 \\
(14.3)\end{array}$ \\
\hline Infection-related deaths, & & & & & & \\
\hline Deaths & 0 & 0 & 0 & $2(0.3)^{*}$ & 0 & 0 \\
\hline
\end{tabular}

alveolar hemorrhage $(n=1)$
Disclosure of Interests: Caroline Gordon Grant/research support from: Sandwell and West Birmingham Hospitals NHS Trust have received funding from UCB to support research work done by my research group that was unrelated to any pharmaceutical product or clinical trial., Consultant for: I have provided consultancy advice and taken part in scientific advisory boards on the design and analysis of clinical trials and the management of lupus for GSK, EMD Serono and UCB. I have taken part in adjudication and safety monitoring committees for BMS., Speakers bureau: I have been paid by UCB for speaking at meetings., Roberto Bassi Employee of: Current employees of EMD Serono, Peter Chang Employee of: Current employees of EMD Serono, Amy Kao Employee of: Current employees of EMD Serono, David Jayne Grant/research support from: David Jayne has received research grants from Chemocentryx, GSK, Roche/Genentech and Sanofi-Genzyme. He has received consultancy fees from Astra-Zeneca, Boehringer-Ingelheim, Chemocentryx, Chugai, GSK, Infla-RX, Insmed and Takeda, David Wofsy Consultant for: GlaxoSmithKline - Member, data safety monitoring board

Novartis - Member, data safety monitoring board

Celgene - member, scientific advisory board, Victor Ona Employee of: Current employees of EMD Serono, Patricia Fleuranceau-Morel Employee of: Current employees of EMD Serono

DOI: 10.1136/annrheumdis-2019-eular.703

\begin{tabular}{|l|l}
\hline FRI0195 & EFFICACY AND SAFETY OF DAPIROLIZUMAB PEGOL \\
(DZP) IN PATIENTS WITH MODERATELY TO SEVERELY \\
ACTIVE SYSTEMIC LUPUS ERYTHEMATOSUS (SLE): A \\
RANDOMISED, PLACEBO (PBO)-CONTROLLED STUDY
\end{tabular}

Richard Furie $^{1}$, lan N. Bruce ${ }^{2}$, Thomas Dörner ${ }^{3}$, Manuel Gustavo Leon ${ }^{4}$, Piotr Leszczynski $^{5}$, Murray B. Urowitz ${ }^{6}$, Birgit Haier ${ }^{7}$, Teri Jimenez ${ }^{8}$, Catherine Barbey ${ }^{9}$, Jiajun Liu ${ }^{10}$, Christian Stach ${ }^{7}{ }^{1}$ Northwell Health, Great Neck, United States of America; ${ }^{2}$ University of Manchester, Manchester, United Kingdom ${ }^{3}$ Charite Berlin, Berlin, Germany; Investigaciones Clínicas, Lima, Peru; ${ }^{5}$ Poznan University of Medical Sciences, Poznan, Poland; ${ }^{6}$ Toronto Western Hospital, Toronto, Canada; ${ }^{7}$ UCB Pharma, Monheim, Germany, ${ }^{8}$ UCB Pharma, Raleigh, United States of America; ${ }^{9}$ Biogen, Baar, Switzerland; ${ }^{10}$ Biogen, Cambridge, United States of America

Background: $C D 40$ ligand (CD40L) regulates interactions between $T$ cells and CD40-expressing cells including antigen-presenting cells (APC) and B cells, thereby playing a critical role in autoimmune disease pathogenesis. DZP, a PEGylated monovalent Fab' antibody fragment with specificity for CD40L, prevents CD40L engagement of CD40 and thus blocks intracellular signalling and APC activation.

Objectives: To report the 24-week efficacy and safety interim data of DZP in a phase Illb, randomised, double-blind, PBO-controlled, dose-ranging study in patients with SLE (NCT02804763).

Methods: Patients with moderately to severely active SLE (SLEDAI-2K score $\geq 6 ; \geq 1$ BILAG grade $A$ or $\geq 2$ BILAG grade $B$ organ domain scores at screening) despite stable non-biologic standard of care treatment were randomised 1:1:1:1 to receive iv DZP 6,24 or $45 \mathrm{mg} / \mathrm{kg}$ or PBO every 4 weeks for 20 weeks. Patients receiving corticosteroids (CS) $\geq 10 \mathrm{mg} /$ day prednisone equivalent were required to start tapering CS by Week 4; guidance was provided, but tapering schedules were ultimately determined by the investigators. The primary objective was to establish a dose-response relationship across three doses of DZP and PBO based on BICLA response rates at Week 24. Four pre-specified dose-response models were tested by a statistical method (MCP-Mod), to determine whether any of the models fit the observed data with statistical significance (at a one-sided $p \leq 0.05$ ). The secondary endpoint was a pairwise comparison of BICLA response rates at Week 24. Other endpoints included the SRI-4 and BICLA response rates at 12 and 24 weeks, mean changes from baseline in SLEDAI-2K scores at 12 and 24 weeks, percentage of patients with daily CS dose $\leq 7.5 \mathrm{mg} /$ day at 12 and 24 weeks, pharmacodynamic (PD) markers and safety.

Results: 182 patients were randomised; $167(91.8 \%)$ completed Week 24 of the study. Baseline demographics were similar across treatment groups. The primary endpoint was not met as none of the pre-specified dose-response models fit the observed BICLA response rates at Week 24 with statistical significance $(p=0.06$ for the most applicable model). BICLA response rates and other efficacy outcome measures at Weeks 12 and 24, were numerically higher for all DZP groups vs PBO (Table 1). Favourable biological effects were observed with improvements in relevant PD markers, including anti-dsDNA antibody levels, in DZP groups vs PBO. Treatment-emergent adverse events (TEAEs) and serious TEAEs were generally balanced across treatment groups (Table 2). More upper 